\title{
In situ detection of lipid peroxidation in chronic hepatitis C: correlation with pathological features
}

\author{
V Paradis, P Mathurin, M Kollinger, F Imbert-Bismut, F Charlotte, A Piton, P Opolon, \\ A Holstege, T Poynard, P Bedossa
}

\begin{abstract}
Aims-To assess the occurrence of lipid peroxidation in chronic hepatitis $\mathbf{C}$ and to evaluate its relation to pathological features and liver iron concentrations.

Methods-Liver biopsy samples of 43 patients with untreated chronic hepatitis C were studied by immunohistochemistry using specific antibodies directed against two major aldehyde metabolites of lipid peroxidation, malondialdehyde (MDA), and 4-hydroxynonenal (HNE).

Results-MDA and HNE adducts (aldehydes covalently linked to another molecule) were detected in the liver samples in $77 \%$ and $30 \%$ of cases, respectively. MDA adducts were detected both in the extracellular matrix and sinusoidal cells localised in areas of periportal and lobular necrosis. HNE adducts appeared in the cytoplasm of only a few hepatocytes. Comparison of the semiquantitative assessment of adducts (MDA and HNE indexes) with the grading and the staging of chronic hepatitis showed that the MDA index was correlated with fibrosis score $(p<0.001)$ and the grade of activity $(p<0.01)$. There was also a tendency to correlation with liver iron concentration $(p=0.09)$. No correlation was observed between the HNE index and pathological features or liver iron concentration.

Conclusion-Lipid peroxidation products are detectable in the liver of chronic hepatitis C patients. The presence of MDA adducts in areas of active fibrogenesis and the correlation between the MDA index and fibrosis score suggest a role for lipid peroxidation in liver fibrosis.

(F Clin Pathol 1997;50:401-406)
\end{abstract}

Keywords: lipid peroxidation; chronic hepatitis C; immunohistochemistry

Department of Pathology

F Charlotte

Department of Internal Medicine I, University of Regensburg, Germany

$M$ Kollinger

A Holstege

Correspondence to

Dr Valérie Paradis, Service

d'Anatomie Pathologique,

Hôpital de Bicêtre, 78, rue

du Général Leclerc, 94275

Le Kremlin-Bicêtre, France.

Accepted for publication

22 January 1997 esis is unclear. Based on data obtained in chronic hepatitis B virus infection, it has been proposed that fibrosis could be the result of necroinflammatory lesions (activity), but the precise mechanisms linking hepatitis $\mathrm{C}$ virus infection, chronic inflammation, and fibrosis have not been determined. ${ }^{2} 3$ Among factors involved in fibrogenesis, cytokines, growth fac- tors, and iron overload have been considered. ${ }^{4-6}$ Furthermore, several recent studies have suggested that lipid peroxidation might play a role in the fibrogenesis observed in some chronic liver diseases..$^{6-8}$ It was therefore proposed that there may be a link between tissue injury and liver fibrosis by modulating collagen gene expression. ${ }^{9-12}$

Lipid peroxidation is caused by free radicals leading to oxidative destruction of polyunsaturated fatty acids constitutive of cellular membranes. ${ }^{13}$ Their destruction leads to the production of toxic and reactive aldehyde metabolites such as malondialdehyde (MDA) and 4-hydroxynonenal (HNE) ${ }^{14}$ These highly cytotoxic metabolites, produced in relatively large amounts, can diffuse from their site of origin to attack distant targets and form covalent links with various molecules (adducts). Therefore, recognition of lipid peroxidation is of interest, as the deleterious effects of this process, including fibrogenesis, might be prevented by administration of scavenging systems or antioxidants. ${ }^{15}$

Most previous studies investigating lipid peroxidation dealt with blood and tissue extracts by indirect quantitative methods; the thiobarbituric acid (TBA) test has been the most commonly applied. ${ }^{16}$ Using this procedure, an increase in MDA was observed in serum and liver of patients with chronic hepatitis C. ${ }^{817}$ Recently, specific antibodies against MDA and HNE adducts have been raised. ${ }^{18} 19$ These antibodies can be used to detect in situ the presence of these lipid peroxidation derived adducts and to specify their cellular localisation.

The aim of this study was to investigate the role of lipid peroxidation in chronic hepatitis $\mathrm{C}$ and its relation with liver fibrosis. To assess the occurrence of lipid peroxidation, MDA and HNE adducts were detected on liver biopsies with an immunohistochemical procedure. The presence of these adducts was then correlated with the grade of activity and the stage of fibrosis assessed on the same biopsy. As iron overload has been reported in chronic hepatitis $\mathrm{C}$, and as this molecule is a cofactor in lipid peroxidation, the presence of these aldehyde adducts was also correlated with liver iron concentration. ${ }^{80}$

\section{Patients and methods}

STUDY GROUP

Forty three patients were studied. All patients were positive for antibody to hepatitis $C$ virus by second generation tests. Serum viral load 
was quantified by branched DNA (second generation bDNA Amplex Chiron, Chiron, Emeryville, California, USA) in 33 patients. Genotype determination was carried out by a competitive oligonucleotide primingpolymerase chain reaction procedure in 32 patients. ${ }^{21}$ All patients were hepatitis $B$ virus negative and had not received any antiviral therapy at least six months before biopsy. Alcohol consumption was recorded in 41 of the 43 patients. Five histologically normal livers in patients without hepatitis $\mathrm{C}$ virus infection were used as controls. These were obtained from surgical resections performed for tumours in an otherwise normal liver.

\section{LIVER BIOPSIES}

Liver biopsy specimens were obtained for each patient using a percutaneous procedure. The liver sample was fixed, paraffin wax embedded, and processed for histological study according to standard procedures (haematoxylin and eosin, Perl's blue, Masson's trichrome). Serial sections were performed for the immunohistochemical study. When enough tissue was available $(n=21)$, the liver iron concentration was determined.

Liver biopsies were examined by two pathologists (VP, PB) in a simultaneous reading. They were blind to the results of lipid peroxidation immunostaining. For each liver biopsy, the semiquantitative assessment of grade of activity and stage of fibrosis according to Metavir was recorded. ${ }^{22}$ The activity of chronic hepatitis was graded as follows: A0, no histological activity; A1, minimal activity; A2, moderate activity; A3, severe activity. The degree of disease activity was defined by the integrated assessment of the major necroinflammatory lesions according to an algorithm previously described. ${ }^{23}$ This grading is based on the semiquantitative assessment of piecemeal necrosis ( 0 , absent; 1 , mild; 2 , moderate; 3 , severe) and lobular necrosis $(0$, absent or mild; 1, moderate; 2, severe). Fibrosis was defined as follows: F0, no fibrosis; F1, portal fibrosis without septa; F2, portal fibrosis with rare septa; F3, numerous septa without cirrhosis; F4, cirrhosis.

\section{IMMUNOHISTOCHEMICAL STUDY}

\section{Antibodies}

Polyvalent monospecific antisera were generated by immunising Watanabe heritable hyperlipidaemic rabbits with homologous low density lipoprotein (LDL) which had been modified in vitro with either $\mathrm{HNE}$ or MDA. LDL $(1.020-1.057 \mathrm{~g} / \mathrm{ml})$ was prepared from $10 \mathrm{ml}$ rabbit blood plasma by sequential ultracentrifugation by using $2.7 \mathrm{mM}$ EDTA, $2 \mathrm{mM}$ benzamidine, $1 \mu \mathrm{M}$ D-phenylalanyl-L-propylL-arginine chloromethyl ketone, $0.01 \%$ aprotinin, $50 \mu \mathrm{g} / \mathrm{ml}$ chloramphenicol, and $100 \mu \mathrm{g} / \mathrm{ml}$ gentamicin to counteract proteolysis. LDL was dialysed against phosphate buffered saline (PBS) containing $0.1 \mathrm{mMEDTA}, 1 \mathrm{mM}$ phenylmethylsulphonylfluoride, and antibiotics.

HNE was generated by acid hydrolysis of hydroxynonenal diethylacetal for one hour at $37^{\circ} \mathrm{C}$. Ten milligrams of purified LDL were incubated with $20 \mathrm{mM}$ (final concentration) $\mathrm{NaCNBH}_{3}, 20 \mathrm{mM}$ EDTA, and $4 \mathrm{mM} \mathrm{HNE}$ in PBS, pH 8.5 for 24 hours at room temperature. After formation of HNE-LDL adducts, excess reagents were removed by extensive dialysis against PBS. The extent of conjugation of lysine residues was $34 \%$, as estimated by the trinitrobenzene sulphonic acid assay. ${ }^{24}$

MDA was generated by acid hydrolysis of MDA-bis-dimethylacetal (Serva, Heidelberg, Germany). MDA-bis-dimethylacetal $(880 \mu \mathrm{l})$ was incubated with $120 \mu \mathrm{l}$ of $4 \mathrm{~N} \mathrm{HCl}$ and $4 \mathrm{ml}$ distilled water for 30 minutes at $37^{\circ} \mathrm{C}$ and titrated to $\mathrm{pH}$ 7.6. Ten milligrams of purified LDL in PBS were incubated with $300 \mu$ of $0.5 \mathrm{M}$ MDA pH 8.5 for three hours at room temperature. After formation of MDA-LDL adducts excess reagents were removed by extensive dialysis against PBS buffer. The extent of conjugation of lysine residues was $78 \%$, as estimated by the trinitrobenzene sulphonic acid assay. ${ }^{24}$

Primary immunisation of Watanabe heritable hyperlipidaemic rabbits consisted of intradermal injections of $200 \mu \mathrm{g}$ of antigen in $500 \mu \mathrm{l}$ PBS suspended with $500 \mu$ l Freund's complete adjuvant. For booster injections, $150 \mu \mathrm{g}$ of antigen were administered in Freund's incomplete adjuvant subcutaneously after two and four weeks. Six weeks after the first immunisation, blood serum was collected. The specificity of antibody was tested by Western blotting using HNE or MDA modified and unmodified proteins. The antibodies were specific for HNE or MDA modified epitopes (adducts).

\section{Immunohistochemical procedure}

The immunohistochemical procedure was performed on serial paraffin wax embedded sections using an automated immunostainer (Techmate 500, Dako, Carpinteria, California, USA) with the avidine-biotin-peroxidase method. For immunohistochemical detection of MDA modified epitopes, slides were pronase treated $(0.01 \%$ in Tris $\mathrm{NaCl}, 25$ minutes) before immunostaining. All primary antibodies were used at a 1:100 dilution.

Localisation and intensity of staining were assessed by the two pathologists in a simultaneous reading, blind to the results of grading and staging. The intensity of the staining (MDA or HNE index) was graded from 0 to 3: 0 , no staining; 1, mild (punctuated labelling); 2, moderate (dense labelling in few cells or in few extracellular foci); 3, strong (dense and homogeneous labelling in many cells or diffuse extracellular staining). This evaluation concerned the mean intensity of the signal on the whole slide. A global lipid peroxidation index was defined by adding the values of MDA and HNE indexes.

Negative controls were normal sheep serum or PBS instead of primary antibodies. Positive controls for lipid peroxidation detection were fixed, paraffin wax embedded rat liver samples from acute hepatitis induced by $\mathrm{CCl}_{4}$. These liver samples were removed 48 hours after an intraperitoneal injection of $100 \mu \mathrm{l} / 100 \mathrm{~g}$ body weight of $\mathrm{CCl}_{4}$ in male Wistar rats. In this 
model, substantial necrosis of centrilobular areas was present 48 hours after $\mathrm{CCl}_{4}$ injection, and it has been shown previously that lipid peroxidation is the major factor responsible for liver necrosis. ${ }^{25}$

\section{LIVER IRON CONCENTRATION}

Liver iron concentration was measured in deparaffinised liver biopsies from 21 patients using the colorimetric assay of Barry and Sherlock. ${ }^{26}$ Results were expressed in $\mu \mathrm{mol} / \mathrm{g}$ of dry weight (dry weight $>0.60 \mathrm{mg}$ for correct chemical iron determination). The normal range (1.2-19.5) was determined in 147 patients with chronic hepatitis $C$ without iron overload using Perl's staining method.

\section{STATISTICAL ANALYSIS}

Quantitative data are presented as mean (SD). Correlations between data were performed using Spearman and Pearson's tests.

\section{Results}

\section{STUDY GROUP}

The study group consisted of 17 women and 26 men, mean age 48 years (range 28-74); 29 patients denied any alcohol consumption. Eleven patients were genotype $1 b$, five were genotype $1 \mathrm{a}$, three were genotype $2 \mathrm{a}$, seven were genotype $3 a$, four were genotype 4 , two were genotype $1 \mathrm{a}+1 \mathrm{~b}$, and one could not be determined. Twenty nine of the 33 patients were hepatitis $C$ virus RNA positive.

Metavir fibrosis grading was as follows: F0, one patient; F1, 18 patients; F2, 14 patients; F3, eight patients; F4, two patients. Six patients had no activity (A0), 15 had mild activity (A1), 11 had moderate activity (A2), and 11 had severe activity (A3).

The mean liver iron concentration determined in 21 patients was 15.1 (14.5) $\mu \mathrm{mol} / \mathrm{g}$ (range 4-71).

\section{IMMUNOHISTOCHEMICAL STUDY}

In rat liver samples with $\mathrm{CCl}_{4}$ induced damage, immunohistochemical study detected strong staining in areas of centrilobular necrosis both with anti-HNE and anti-MDA antibodies (data not shown). No staining was observed in portal tracts and periportal areas. In control experiments, where non-immune serum was used instead of anti-adduct antibody, no staining was observed.

MDA or HNE adducts or both were detected immunohistochemically in 35 of 43 patients $(81 \%)$. MDA adducts were detected in 33 of the 43 patients (77\%). Staining was graded mild in 23 cases, moderate in nine cases, and strong in one case. Immunostaining was observed both in the extracellular matrix and sinusoidal cells. When staining was mild, it was restricted to the periportal areas (fig 1A). In cases of moderate or strong staining, a more diffuse portal labelling was observed, displaying a thin extracellular network (fig 1B). In these cases, patches of lobular staining were also present, localised in the sinusoidal cells in contact with lobular necrosis foci (fig 1C). No staining was observed in hepatocytes.
HNE adducts were detected in 13 of the 43 cases $(30 \%)$. Ten of the 13 cases $(77 \%)$ that displayed HNE adducts also showed MDA adducts. The staining was mild in 12 cases and moderate in one case. It was mainly intracellular and appeared in the form of small granules or vesicles in the cytoplasm of hepatocytes (fig 1D). No specific topographical distribution of labelling was observed and immunostaining was not co-localised with lobular or periportal necrosis.

The five histologically normal livers of control patients showed no labelling with anti$\mathrm{HNE}$ antibody. Mild extracellular staining was observed in portal tracts with anti-MDA antibody.

CORRELATION BETWEEN LIPID PEROXIDATION AND BIOLOGICAL AND HISTOLOGICAL DATA

There was a significant correlation between the presence and the intensity of MDA adducts in the liver and both the grade of activity $(p<0.01)$ and the stage of fibrosis $(\mathrm{p}<0.001)$. No correlation was observed between the HNE index and the grade of activity or the stage of fibrosis. The lipid peroxidation index was significantly correlated only with the stage of fibrosis $(p<0.01)$. The values of MDA, HNE, and lipid peroxidation indexes according to the grade of activity or the stage of fibrosis are reported in table 1 .

MDA and HNE indexes were not significantly different in the group of patients with genotype $1 \mathrm{~b}$ versus patients with other genotypes (MDA $0.92(0.9) v 1.15$ (0.56); HNE $0.23(0.57) v 0.37(0.49))$. There was no correlation between MDA, HNE, or lipid peroxidation indexes and age or sex of the patients. Although lipid peroxidation, MDA, and HNE indexes were higher in alcohol consumers than in abstinent patients, these differences were not statistically significant (lipid peroxidation 1.75 (1.29) $v 1.14$ (0.88); MDA $1.25(0.87) v 0.90$ (0.67); HNE $0.50(0.67) v 0.28(0.45)$ ).

Although not significant, there was a tendency to correlation between MDA index and liver iron concentration $(p=0.09)$. The liver iron concentration was 10.6 (3.2) $\mu \mathrm{mol} / \mathrm{g}$ in the 14 patients with an MDA index of 0 or 1 , and $17.4(17.5) \mu \mathrm{mol} / \mathrm{g}$ in the seven patients with an MDA index of 2 . There was no correlation between HNE index and liver iron concentration (14.6 (7.6) $\mu \mathrm{mol} / \mathrm{g}$ in patients with an HNE index of 0 compared with 15.9 (22.4) $\mu \mathrm{mol} / \mathrm{g}$ in patients with an HNE index $>0)$. No correlation was observed between lipid peroxidation index and liver iron concentration $(14.2(8.9) \mu \mathrm{mol} / \mathrm{g}$ in patients with a lipid peroxidation index of 0 or 1 compared with $16.1(19.5) \mu \mathrm{mol} / \mathrm{g}$ in patients with a lipid peroxidation index $>1$ ). Furthermore, liver iron concentration was correlated neither with the grade of activity nor the stage of fibrosis.

\section{Discussion}

Lipid peroxidation, a free radical induced mechanism, is implicated in the pathogenesis of several acute and chronic human disorders, including liver pathology. ${ }^{6-8} 27$ The deleterious consequences of this mechanism are related in 

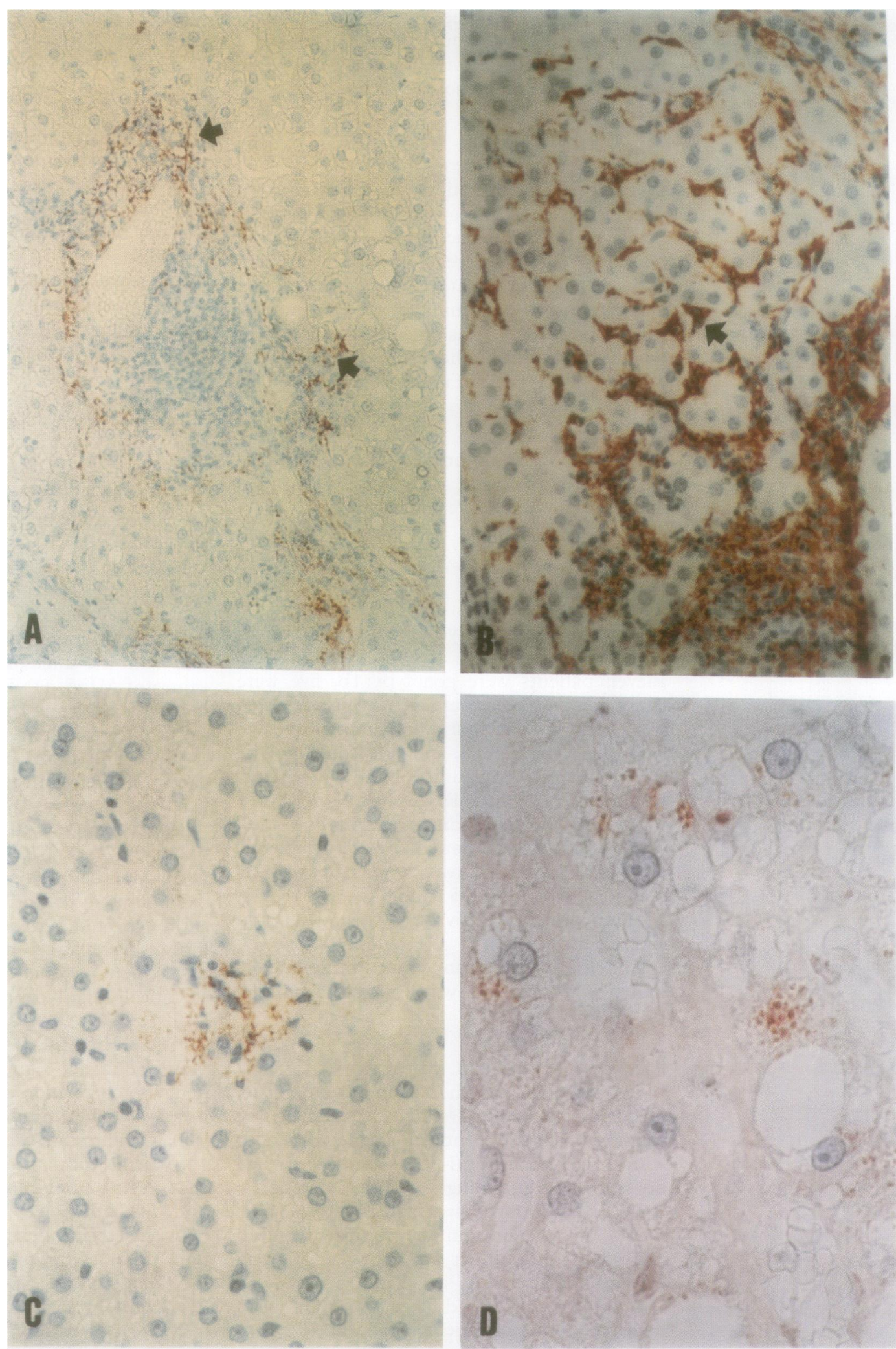

Figure 1 Immunohistochemical detection of malondialdehyde (MDA) and 4-hydroxynonenal (HNE) in chronic hepatitis $C$. (A) Immunostaining of MDA adducts in chronic hepatitis $C$ with mild activity (A1) and portal fibrosis (F1). $A$ moderate MDA adduct immunostaining (grade 2) is observed in the periportal extracellular matrix (arrow). The centre of the portal tract is not labelled (magnification $\times 25$ ). (B) Immunostaining of MDA adducts in chronic hepatitis $C$ with severe activity (A3). A strong $M D A$ adduct immunostaining (grade 3) is detected in periportal areas both in the extracellular matrix and in sinusoidal cells (arrow) (magnification $\times 100$ ). (C) Immunostaining of MDA adducts in chronic hepatitis $C$ with lobular necrosis. MDA adducts are observed in sinusoidal cells located in an area of lobular necrosis (mild staining, grade 1) (magnification $\times 200)$. (D) Immunostaining of $H N E$ adducts in chronic hepatitis $C$. A mild intracellular HNE adduct immunostaining (grade 1) is detected in the cytoplasm of a few hepatocytes in the form of either small granules close to steatosis vacuoles or intracytoplasmic vesicles (magnification $\times 400)$.

part to the formation of aldehyde products such as MDA and HNE that bind to various molecules, impairing their functions. ${ }^{14}$

We investigated the presence of MDA and
HNE adducts in liver samples from patients with chronic hepatitis $\mathrm{C}$ virus infection by an immunohistochemical procedure. Using this technique, we detected at least one marker of 
Table 1 Malondialdehyde (MDA), 4-hydroxynonenal (HNE), and lipid peroxidation indexes in patients with chronic hepatitis $C$ according to the grade of activity $(A)$ and the stage of fibrosis $(F)^{22}$

\begin{tabular}{llll}
\hline & & & \\
& $M D A$ index & HNE index & $\begin{array}{l}\text { Lipid } \\
\text { peroxidation } \\
\text { index }\end{array}$ \\
\hline Activity & & & \\
A0 or A1 (n=21) & $0.76(0.62)$ & $0.19(0.4)$ & $0.95(0.4)$ \\
A2 (n=11) & $1.00(0.88)$ & $0.55(0.5)$ & $1.03(1)$ \\
A3 (n=11) & $1.55(0.52)$ & $0.36(0.67)$ & $1.04(1)$ \\
p $^{\star}$ & $<0.01$ & NS & NS \\
Fibrosis & & & $1.0(1.03)$ \\
F0 or F1 (n=19) & $0.65(0.67)$ & $0.31(0.48)$ & $1.5(0.94)$ \\
F2 (n=14) & $1.07(0.53)$ & $0.36(0.63)$ & $1.8(0.94)$ \\
F3 or F4 (n=10) & $1.67(0.45)$ & $0.30(0.48)$ & 1.01 \\
p $^{\star}$ & $<0.001$ & NS & $<0.01$ \\
\hline
\end{tabular}

Values are mean (SD). *Spearman and Pearson's tests.

lipid peroxidation in $81 \%$ of patients infected by hepatitis $C$ virus. These results are concordant with previous studies performed on blood or tissue extracts, and suggest that lipid peroxidation occurs in chronic hepatitis C. ${ }^{817}$ Using immunohistochemistry, we were also able to elucidate the tissular and cellular localisation of these aldehyde products in relation to pathological features.

This procedure revealed that MDA adducts are the major detectable aldehyde adducts in the liver, and the intensity of staining is correlated with the severity of chronic hepatitis. Although MDA and HNE are both products of lipid peroxidation, we observed that, for a given case, the intensity and localisation of MDA and HNE adducts were different. It is of note that production of lipid peroxidation byproducts does not imply their in situ immunohistochemical detection. In fact, we used antibodies that detect only MDA or HNE adducts but not free HNE and MDA. In order to be detected, these products have to be immobilised onto the tissue by binding to intracellular or extracellular molecules, a mechanism which differs according to the nature of the lipid peroxidation byproducts. MDA is a dialdehyde of short half life that reacts preferentially with amino groups of proteins, inducing the formation of intermolecular and intramolecular bridging. In contrast, HNE reacts with thiol groups ${ }^{14}$ and has a relatively long half life. The different immunostaining patterns observed between MDA and HNE reflect both their different biochemical properties and their different half lives.

The correlation between MDA index and the grade of activity of chronic hepatitis suggests that lipid peroxidation is involved in the pathogenesis of the necroinflammatory reaction. However, whether lipid peroxidation is the cause or the consequence of the liver damage remains to be elucidated. The presence of MDA adducts in occasional cases with no or mild activity may suggest a causative mechanism.

In vitro studies have shown that lipid peroxidation products stimulate collagen production by regulating collagen gene transcription in fibroblasts and hepatic stellate cells. ${ }^{9}$ In chronic hepatitis C virus infection, we observed a strong association between the MDA index or the lipid peroxidation index and the stage of fibrosis. Furthermore, MDA adducts were preferentially detected in sinusoidal cells in close contact with areas of active fibrogenesis. These data suggest a causative relation between lipid peroxidation and liver fibrosis in patients with chronic hepatitis $\mathrm{C}$ virus infection.

In chronic hepatitis $\mathrm{C}$, iron overload has been noted in some studies. ${ }^{8}$ Although slight, this overload has been evoked as a factor of resistance to interferon therapy. ${ }^{28}{ }^{29}$ This is of interest as iron exerts its cytotoxic effects through enhanced formation of free radicals and lipid peroxidation stimulation. Although we observed a slight increase in liver iron concentration in our patients, a tendency to correlation was noted between liver iron concentration and the MDA index. If these results are confirmed in a larger samples of patients, it could be postulated that enhanced lipid peroxidation observed in chronic hepatitis $\mathrm{C}$ is linked in part to iron overload.

In conclusion, this study shows that lipid peroxidation products can be detected in liver biopsies of patients with chronic hepatitis $\mathrm{C}$ virus infection. There is experimental evidence showing that lipid peroxidation can be prevented by administration of scavengers of free radicals or antioxidants. ${ }^{15}$ It has been shown that lipid peroxidation products can stimulate fibrogenesis by inducing collagen gene expression thus, detection and prevention of lipid peroxidation could be of major interest in preventing fibrosis and cirrhosis in this disease.

This study was supported by a grant from the Association pour la Recherche supported by a grant from the Association pour Forschungs Gemeinshaft. Hydroxynenal diethylacetal was kindly provided by $H$ Esterbauer and $G$ Waeg, University of kraz, Austria.

1 Soni P, Dusheiko GM, Harrison TI, Dhillon AP Genetic Soni P, Dusheiko GM, Harrison TJ, Dhillon AP. Genetic diversity of hepatitis C virus: implications for pathogen

2 Liaw Y-F, Tai D-I, Chu C-M, Chen T-J. The development of cirrhosis in patients with chronic type B hepatitis: a prospective study. Hepatology 1988;8:493-6.

3 Cooksley WG, Bradbear RA, Robinson W, Harrison M, Halliday JW, Powell LW, et al. The prognosis of chronic active hepatitis without cirrhosis in relation to bridging necrosis. Hepatology 1986;6:345-8.

4 Zern MA, Czaja MJ, Weiner FR. The use of molecular hybridization techniques as tools to evaluate hepatic fibrogenesis. In: Rojkind $\mathrm{M}$, ed. Connective tissue in health and gisease. Boca Raton: CRC Press, 1990:99-122.

5 Bachem M, Meyer D, Melchior R, Sell KM, Gressner AM Activation of rat liver perisinusoidal lipocytes by transforming growth factors derived from myofibroblast-like cells. A potential mechanism of self perpetuation in liver fibrogenesis. F Clin Invest 1992;89:19-27.

6 Farinati F, Cardin R, De Maria N, Lecis PE, Della Libera $G$, Burra $P$, et al. Zinc, iron and peroxidation in liver tissue. Biol Trace Elem Res 1995;47:193-9.

7 Britton RS, Bacon BR. Role of free radicals in liver diseases and hepatic fibrosis. Hepatogastroenterology 1994;41:343-8.

8 Farinati F, Cardin R, De Maria N, Della Libera G, Marafin C, Lecis E, et al. Iron storage, lipid peroxidation and glutathione turnover in chronic anti-HCV positive hepatiglutathione turnover in chronic
tis. $\mathcal{F}$ Hepatol $1995 ; 22: 449-56$.

9 Parola M, Pinzani M, Casini A, Albano E, Poli G, Gentilini A, et al. Stimulation of lipid peroxidation or 4-hydroxynonenal treatment increases procollagen $\alpha 1$ (I) gene expression in human fat-storing cells. Biochem Biophys Res Comm 1993;194:1044-50.

10 Kamimura S, Gaal K, Britton RS, Bacon BR, Triadafilopoulos $G$, Tsukamoto $H$. Increased 4-hydroxynonenal levels in experimental alcoholic liver disease: association of lipid peroxidation with liver fibrogenesis. Hepatology 1992 16:448-53.

11 Chojkier M, Houglum K, Herruzo JS, Brenner DA. Stimulation of collagen expression by ascorbic acid in cultured human fibroblasts. F Biol Chem 1989;264:16957-62.

12 Bedossa P, Houglum K, Trautwein C, Holstege A, Alcorn J, Chojkier M. Stimulation of collagen $\alpha 1$ (I) gene expression is associated with lipid peroxidation in hepatocellular injury: a associated with lipid peroxidation in hepatocellular
link to tissue fibrosis? Hepatology 1994;19:1262-71.

13 Cheeseman KH. Mechanisms and effects of lipid peroxidation. Molec Aspects Med 1993;14:191-7. 
14 Esterbauer H, Schaur RJ, Zollner H. Chemistry and biochemistry of 4-hydroxynonenal, malonaldehyde and related aldehydes. Free Rad Biol Med 1992;11:81-128.

15 Poli G, Parola M, Leonarduzzi G, Pinzani M. Modulation of hepatic fibrogenesis by antioxidants. Molec Aspects Med 1993;14:259-64.

16 Janero DR. Malondialdehyde and thiobarbituric acidreactivity as diagnostic indices of lipid peroxidation and peroxidative tissue injury. Free Rad Biol Med 1990;9:51540.

17 Farinati F, Cardin R, D'Errico A, De Maria N, Naccarato $\mathrm{R}$, Cecchetto A, et al. Hepatocyte proliferative activity in chronic liver damage as as by the monoclona antibody MIB1 Ki67 in archival material: the role of etiology, disease activity, iron, and lipid peroxidation. Hepatol ogy 1996;23:1468-75.

18 Uchida K, Szweda LI, Chae H-Z, Stadtman ER. Immunochemical detection of 4-hydroxynonenal protein adducts in oxidized hepatocytes. Proc Natl Acad Sci USA 1993;90: 8742-6.

19 Palinski W, Yla-Herttualaa S, Rosenfeld ME, Butler SW, Socher SA, Parthasanthy S, et al. Antisera and monoclona antibodies specific for epitopes generated during oxidative modification of low density protein. Arteriosclerosis 1990; 10:325-35.

20 Olynyk JK, O’Neill R, Britton RS, Bacon BR. Determination of hepatic iron concentration in fresh and paraffinembeddded tissue: diagnostic implications. Gastroenterology 1994;106:674-7.
21 Sajus M, Olivi M, Vidaud D, Mathurin P, Opolon P, Poynard $\mathrm{T}$, et al. Analysis of $\mathrm{HCV}$ genotypes by a fluorescent competitive oligonucleotide priming PCR (COP-PCR) [abstract]. Hepatology 1994;20:244a.

22 The METAVIR cooperative group. Inter- and intraobserver variation in the assessment of liver biopsy of chronic hepatitis C. Hepatology 1994;20:15-20.

23 Bedossa P, Poynard T for the METAVIR cooperative study group. An algorithm for the grading of activity in chronic group. An algorithm for the grading of
hepatitis C. Hepatology 1996;24:289-93.

24 Habeeb AFSA. Determination of free amino groups in proteins by trinitrobenzenesulfonic acid. Anal Biochem 1966; 14:328-36.

25 Recknagel RO, Glende EA, Dolak JA. Mechanisms of carbon tetrachloride toxicity. Pharmacol Therapy 1987;43: 139-54.

26 Barry M, Sherlock S. Measurement of liver iron concentration in needle-biopsy specimens. Lancet 1971;i:100-3.

27 Poli G. Liver damage due to free radicals. Br Med Bull 1993; 49:604-20.

28 Higueras V, Raya A, Rodrigo JM, Serra MA, Roma J, Romero FJ. Interferon decreases serum lipid peroxidation products of hepatitis C patients. Free Rad Biol Med 1994;16:131-3.

29 Olynyk JK, Di Bisceglie AM, Jeffers LJ, Parker TI, Radick $\mathrm{JL}$, Schiff ER, et al. Hepatic iron concentration as a predictor of response to interferon alfa therapy in chronic hepatitis C. Gastroenterology 1995;108:1104-9. 